\title{
A GENERALIZATION OF THE ISOPERIMETRIC INEQUALITY
}

\author{
THOMAS F. BANCHOFF \& WILLIAM F. POHL
}

1. For a simple closed plane curve of length $L$ bounding an area $A$ the classical isoperimetric inequality asserts that

$$
L^{2}-4 \pi A \geq 0
$$

with equality holding only for a circle. We show here that this inequality remains true for non-simple closed curves where in place of $A$ we take the sum of the areas into which the curve divides the plane, each weighted with the square of the winding number, i.e.,

$$
L^{2}-4 \pi \int_{E^{2}} w^{2} d A \geq 0,
$$

where, for $p \in E^{2}, w(p)$ is the winding number of $p$ with respect to the curve. Equality holds if and only if the curve is a circle, or a circle traversed several times or several coincident circles each traversed in the same direction any number of times. Note that this implies that

$$
L^{2}-4 \pi \int_{E^{2}}|w|^{p} d A \geq 0
$$

for any $0<p \leq 2$ and that 2 is here the best possible power.

This may all be generalized to arbitrary dimension and codimension. For the case of closed space curves let $G$ denote the space of lines in $E^{3}$ (parallel lines are not identified) and let $d G$ denote its invariant measure [1], [7]. Then

$$
L^{2}-4 \int_{G} \lambda^{2} d G \geq 0,
$$

where $\lambda(l)$ denotes the linking number of $l \in G$ with the curve. Equality holds

Received February 14, 1970. The work of the first author was supported by the National Science Foundation under Grant GP-7610, and that of the second author by the N.S.F. under Grant GP-5760 and the Netherlands Organization for the Advancement of Pure Research (Z.W.O.). 
here only for one or several coincident circles, as before. For closed oriented surfaces $M$ in $E^{3}$ we obtain

$$
\int_{M \times M} r^{-1} d A_{1} d A_{2}-12 \pi \int_{E^{3}} w^{2} d V \geq 0,
$$

where, for $(x, y) \in M \times M, r$ is the length of the chord joining $x$ and $y, d A_{1}$ and $d A_{2}$ denote the elements of area of $M$ at $x$ and $y$, respectively, and $w(p), p \in E^{3}$, denotes the winding number of the surface with respect to $p$. Equality holds only for one or several coincident spheres with coincident orientations. Note that the left-hand integral is essentially the gravitational self-potential of $M$ considered as a thin homogeneous shell. The right-hand integral is just the volume in case $M$ is connected and embedded.

The general result is as follows. Let $M$ be a compact oriented manifold of dimension $m$, and $f: M \rightarrow E^{n}$ be an immersion of class $C^{2}$. For $(x, y) \in M \times M$ let $r(x, y)$ denote the chord length from $f(x)$ to $f(y)$, and let $d V_{1}, d V_{2}$ denote the volume elements on $M$ at $x$ and $y$ respectively. Let $H_{n-m-1, n}$ denote the Grassmann manifold of $(n-m-1)$-planes in $E^{n}$ (parallel planes are not identified), and let $\left|d H_{n-m-1, n}\right|$ denote its invariant measure [1], [2], [6], [7]. For $h \in H_{n-m-1, n}$ let $\pm \lambda$ denote the linking number of $h$ with $f$.

Theorem 1.

$$
\int_{M \times M} r^{-m+1} d V_{1} d V_{2}-(1+m) \Sigma_{m} K_{m, n} \int_{H_{n-m-1, n}} \lambda^{2}\left|d H_{n-m-1, n}\right| \geq 0,
$$

where $\Sigma_{m}$ denotes the surface volume of the unit $m$-sphere and $K_{m, n}$ is a constant which depends only on $m$ and $n$. Equality holds only for one or several coincident spheres with coincident orientations, or $(n=1)$ one or several coincident circles all traversed in the same direction each a number of times.

Let us take the second integral in this inequality and write

$$
\mathscr{A}(M)=K_{m, n} \int_{H_{n-m-1}, n} \lambda^{2}\left|d H_{n-m-1, n}\right| .
$$

Then $\mathscr{A}(M)$ may be thought of, in the case of a space curve, as the "area" bounded by the curve, or in general as the "volume" bounded by a submanifold of higher codimension of a euclidean space. There are, of course, several candidates for such a "volume", e.g., the surface volume of the submanifold of dimension $m+1$ of least surface volume spanning $f(M)$, or the volume of the convex hull, or the surface volume of the convex envelope. $\mathscr{A}(M)$, however, has the following simple properties:

1) $\mathscr{A}(M)$ is just the volume bounded by $f(M)$ if $f$ is an embedding into a linear space of dimension $m+1$; 
2) $\mathscr{A}(M)$ is stable under raising of the codimension, i.e., if $M \subset E^{n} \subset E^{N}$, then $\mathscr{A}(M)$ in the sense of submanifolds of $E^{N}$ is the same as $\mathscr{A}(M)$ in the sense of submanifolds of $E^{n}$;

3) $\mathscr{A}(M)$ is finite and is given by an integral over $M \times M$ (cf. Theorem 4 and the Remark following);

4) $\mathscr{A}(M)$ has the "reproductive" property in the sense of Chern [3], i.e., for $q>n-m-1, H_{q} \in H_{q, n}$, we have

$$
l_{m, n, q} \int_{H_{q}, n} \mathscr{A}\left(M \cap H_{q}\right)\left|d H_{q, n}\right|=\mathscr{A}(M),
$$

where $l_{m, n, q}$ is a constant depending only on $m, n$ and $q$ (cf. Theorem 3);

5) $\mathscr{A}(M)$ satisfies an "isoperimetric" inequality (Theorem 1 above).

This paper is organized as follows. In $\S 2$ we prove a theorem on the convergence of certain sequences of integrals which we shall use in the sequel; in $\S 3$ we establish properties 1 ) -4) above; the proof of Theorem 1 is given in $\S 4$; and in $\S 5$ we prove some additional inequalities using the same methods. Some of these seem to be new even for convex surfaces. $\S \S 3$ and 4 are based on [6]; however, we give explicit references, so that the present paper may be read without first reading [6], the reader looking up the references as needed. Theorem 3 was proved in a special case in [6, Formula 1]. Our proof here is considerably simpler.

Theorem 1 for the case of plane curves is much simpler to prove than the general result and is suitable for presentation in an elementary course. To extract this simpler proof one uses Theorem 3 in the special case $q=m=1$, $n=2$, where it is quite easy to prove; Theorem 4 which is easy to prove in this case, since in the plane $d I$ is essentially the invariant measure for lines; Proposition 5, which is given here a separate simple proof for curves; the local analysis proceeding Proposition 5, which is also done separately for curves; and, finally, the proof of Theorem 1 as it stands in $\S 4 . \S 2$ is not needed, and a simpler account of $S(M)$ is given in [5].

Our proof of Theorem 1 generalizes one given for plane convex curves by Arne Pleijel [4]. We wish to thank H.Guggenheimer for bringing the work of Pleijel to our attention. We also wish to thank Mario Miranda for help with the proof of Proposition 2.

2. In this section we establish convergence of certain limits of integrals in case $m>1$. Let $M^{m}=M$ denote a compact differentiable manifold of dimension $m$, and let $f_{k}: M \rightarrow E^{n}, k=1,2, \cdots$, denote a sequence of $C^{1}$ immersions which converge uniformly to an immersion $f$ such that the first derivatives of $f_{k}$ converge uniformly to the first derivatives of $f$. Let $d V_{k}$ and $d V$ denote the volume elements of $f_{k}$ and $f$ respectively. Let $\pi_{1}, \pi_{2}: M \times M \rightarrow M$ denote the projection mappings into the first and second factors, respectively, and let 
$d V_{k i}=\pi_{i}^{*} d V_{k}, d V_{i}=\pi_{i}^{*} d V, i=1,2, r_{k}(x, y)=\left|f_{k}(y)-f_{k}(x)\right|$ and $r(x, y)$ $=|f(y)-f(x)|$.

Proposition 2. Let $\left\{\Phi_{k}\right\}$ denote a sequence of real-valued functions on $M \times M$ such that $\left|\Phi_{k}\right| \leq 1$ and

$$
\lim _{k \rightarrow \infty} \Phi_{k}=\Phi \quad \text { a.e. }
$$

Then

$$
\lim _{k \rightarrow \infty} \int_{M \times M} \Phi_{k} r_{k}^{-m+1} d V_{k 1} d V_{k 2}=\int_{M \times M} \Phi r^{-m+1} d V_{1} d V_{2}
$$

and all these integrals are absolutely convergent.

Proof. Let $P_{1}$ and $P_{2}$ be two $m$-planes in $R^{n}$, and define the angle $\theta=$ $\angle\left(P_{1}, P_{2}\right)$ between them as follows. The metric in $R^{n}$ induces a metric in $\wedge^{m} R^{n}$ which with suitable normalization is given by the formula $e_{1} \wedge \cdots \wedge e_{m} \cdot f_{1} \wedge$ $\cdots \wedge f_{m}=\operatorname{det}\left[e_{i} \cdot f_{j}\right]$. If $e_{1}, \cdots, e_{m}$ is an orthonormal set of vectors spanning $P_{1}$, and $f_{1}, \cdots, f_{m}$ an orthonormal set of vectors spanning $P_{2}$, then we define $\theta$ by $\cos \theta=\left|e_{1} \wedge \cdots \wedge e_{m} \cdot f_{1} \wedge \cdots \wedge f_{m}\right|$ and $0 \leq \theta \leq \pi / 2$. We may interpret this as follows. $e_{1} \wedge \cdots \wedge e_{m}$ and $f_{1} \wedge \cdots \wedge f_{m}$ have unit norm and hence represent points on the unit sphere in $\wedge^{m} R^{n}$. If these points are joined with the origin by lines, then $\theta$ is the lesser positive angle between these lines and can be measured as a distance on the unit sphere in $\wedge^{m} R^{n}$.

We assert that there is a real number $\varepsilon>0$ such that for any pair of $m$-planes $P_{1}$ and $P_{2}$ in $E^{n}$ there exists an $m$-plane $Q$ such that $\angle\left(P_{1}, Q\right) \leq \pi / 2-\varepsilon$ and $\angle\left(P_{2}, Q\right) \leq \pi / 2-\varepsilon$. To show this it suffices to consider only $m$-planes through the origin, which form a compact Grassmann manifold $G_{m, n}$, since $\theta$ is unchanged by parallel translation of $P_{1}$ and $P_{2}$. Suppose the assertion is false. Then there exist sequences of $m$-planes $\left\{P_{1 n}\right\},\left\{P_{2 n}\right\}$ such that for any $n$-plane $Q$ either $\angle\left(P_{1 n}, Q\right)>\pi / 2-1 / n$ or $\angle\left(P_{2 n}, Q\right)>\pi / 2-1 / n$. We may extract subsequences from $\left\{P_{1 n}\right\}$ and $\left\{P_{2 n}\right\}$ which converge to $P_{1 \infty}$ and $P_{2 \infty}$ respectively, and these have the property that for any $m$-plane $Q$, either $\angle\left(P_{1 \infty}, Q\right)=\pi / 2$ or $\angle\left(P_{2 \infty}, Q\right)=\pi / 2$. But this is impossible since $G_{m, n}$ is an irreducible algebraic variety, and the assertion is proven.

For $(x, z) \in M \times M$ let $d_{k}(x, z), d(x, z)$ denote the distance between $x$ and $z$ in the sense of the Riemannian metric induced on $M$ by $f_{k}$ and $f$, respectively, and $T_{x k}, T_{x}$ the tangent $m$-planes at $x$ to $f_{k}$ and $f$ respectively. Since $f$ is differentiable of class $C^{1}$ and $M$ is compact, we may find a $\delta>0$ such that if $d(x, z)<\delta$, then $\angle\left(T_{x}, T_{z}\right)<\varepsilon / 2$. By the uniform convergence of the derivatives we may find a positive integer $K$ such that if $k>K$ then $\angle\left(T_{z k}, T_{z}\right)<\varepsilon / 4$ for all $z$, so that if $d(x, z)<\delta$ then

$$
\angle\left(T_{z k}, T_{x}\right) \leq \angle\left(T_{z k}, T_{z}\right)+\angle\left(T_{z}, T_{x}\right)<3 \varepsilon / 4 .
$$


On $M \times M$ let us take the metric $D\left(\left(x_{1}, y_{1}\right),\left(x_{2}, y_{2}\right)\right)=\left(d\left(x_{1}, x_{2}\right)^{2}+d\left(y_{1}, y_{2}\right)^{2}\right)^{1 / 2}$. Let us now take a partition of unity $\sum \varphi_{\alpha}=1$ on $M \times M$, whose supports are contained in open sets $U_{1}, \cdots, U_{N}$ of diameter $<\delta$ is the metric $D$, and for each $i$ choose a point $\left(x_{i}, y_{i}\right) \in U_{i}$ and an $m$-plane $Q_{i}$ such that

$$
\angle\left(T_{x_{i}}, Q_{i}\right)<\pi / 2-\varepsilon \text { and } \angle\left(T_{y_{i}}, Q_{i}\right)<\pi / 2-\varepsilon .
$$

If $(x, y) \in U_{i}$, then

$$
\begin{aligned}
& \angle\left(Q_{i}, T_{x k}\right) \leq \angle\left(Q_{i}, T_{x_{i}}\right)+\angle\left(T_{x_{i}}, T_{x k}\right) \leq \pi / 2-\varepsilon / 4, \\
& \angle\left(Q_{i}, T_{y k}\right) \leq \angle\left(Q_{i}, T_{y_{i}}\right)+\angle\left(T_{y_{i}}, T_{y k}\right) \leq \pi / 2-\varepsilon / 4 .
\end{aligned}
$$

Let $E=\sec (\pi / 2-\varepsilon / 4)$. It follows that we can represent $f_{k}\left(\pi_{1}\left(U_{i}\right)\right)$ and $f_{k}\left(\pi_{2}\left(U_{i}\right)\right)$ non-parametrically in $E^{n}$ with $Q_{i}$ as base plane; i.e., if we take Cartesian coordinates $x_{1}, \cdots, x_{n}$ in $E^{n}$ such that $Q_{i}$ is defined by $x_{m+1}=\cdots$ $=x_{n}=0$, then we may represent $f_{k}\left(\pi_{1}\left(U_{i}\right)\right)$ and $f_{k}\left(\pi_{2}\left(U_{i}\right)\right)$ as

$$
\begin{array}{cc}
x_{m+1}=f_{m+1}^{k}\left(x_{1}, \cdots, x_{m}\right), & x_{m+1}=\bar{f}_{m+1}^{k}\left(x_{1}, \cdots, x_{m}\right), \\
\vdots & \vdots \\
x_{n}=f_{n}^{k}\left(x_{1}, \cdots, x_{m}\right), & x_{n}=\dot{f}_{n}^{k}\left(x_{1}, \cdots, x_{m}\right),
\end{array}
$$

respectively, and similarly for $f\left(\pi_{1}\left(U_{i}\right)\right)$ and $f\left(\pi_{2}\left(U_{i}\right)\right)$.

Let $q_{1}, q_{2}: Q_{i} \times Q_{i} \rightarrow Q_{i}$ denote the projections into the first and second factor, respectively, and let $y_{i}=x_{i} \circ q_{1}, z_{i}=x_{i} \circ q_{2}$. Let $p: E^{n} \rightarrow Q_{i}$ denote orthogonal projection and let

$$
C_{k}=\left(p \circ f_{k} \circ \pi_{1}\right) \times\left(p \circ f_{k} \circ \pi_{2}\right): U_{i} \rightarrow Q_{i} \times Q_{i} .
$$

Let $\chi_{k}$ denote the characteristic function of $C_{k}\left(U_{i}\right)$, so that

$$
\chi_{k}(p)=\left\{\begin{array}{l}
1, p \in C_{k}\left(U_{i}\right) \\
0, p \in Q_{i} \times Q_{i}-C_{k}\left(U_{i}\right)
\end{array}\right.
$$

Let $U_{i}^{\prime}=\cup_{k} C_{k}\left(U_{i}\right)$, and let $\chi$ denote its characteristic function. We can choose $K^{\prime}$ so large that if $k>K^{\prime}$ then the Euclidean distance between $f(x)$ and $f_{k}(x)$ is less than $\delta / 2$ for all $x$. We henceforth assume $k>K^{\prime}$. Then the diameter of $U_{i}^{\prime}$ is less than $4 \delta$, and

$$
\begin{aligned}
& \int_{U_{i}} \varphi_{i}\left|\Phi_{k}\right| r_{k}^{-m+1} d V_{k 1} d V_{k 2} \\
&=\int_{Q_{i} \times Q_{i}} \chi_{k}\left[\varphi_{i}\left|\Phi_{k}\right| r_{k}^{-m+1}\right] \circ C_{k}^{-1} \sec \angle\left(T_{k}, Q_{i}\right) \sec \angle\left(\bar{T}_{k}, Q_{i}\right) d y_{1} \\
& \cdots d y_{m} d z_{1} \cdots d z_{m},
\end{aligned}
$$


where $T_{k}(p)=T_{q k}, q=\pi_{1} C_{k}^{-1}(p)$, and $\bar{T}_{k}(p)=T_{s k}, s=\pi_{2} C_{k}^{-1}(p)$. Now for $p$ a variable point in $Q_{i} \times Q_{i}$

$$
\begin{gathered}
\chi_{k}\left[\varphi_{i}\left|\Phi_{k}\right| r_{k}^{-m+1}\right] \circ C_{k}^{-1} \sec \angle\left(T_{k}, Q_{i}\right) \sec \angle\left(\bar{T}_{k}, Q_{i}\right) \\
\leq \chi\left(\sum_{j=1}^{m}\left(y_{j}-z_{j}\right)^{2}{ }^{(-m+1) / 2} E^{2}\right. \\
\int_{Q_{i} \times Q_{i}} \chi\left(\sum_{j=1}^{m}\left(y_{j}-z_{j}\right)^{2}\right)^{(-m+1) / 2} E^{2} d y_{1} \cdots d y_{m} d z_{1} \cdots d z_{m} \leq \Sigma_{m-1}(4 \delta)^{m+1} E^{2},
\end{gathered}
$$

where $\Sigma_{m-1}$ is the surface volume of the unit $(m-1)$-sphere, as is shown by a standard computation using polar coordinates. Thus by the Lebesgue bounded convergence theorem

$$
\lim _{k \rightarrow \infty} \int_{U_{i}} \varphi_{i} \Phi_{k} r_{k}{ }^{-m+1} d V_{k 1} d V_{k 2}=\int_{U_{i}} \varphi_{i} \Phi r^{-m+1} d V_{1} d V_{2},
$$

and all these integrals are absolutely convergent. Hence

$$
\begin{gathered}
\lim _{k \rightarrow \infty} \int_{M \times M} \Phi_{k} r_{k}^{-m+1} d V_{k 1} d V_{k 2}=\lim _{k \rightarrow \infty} \sum_{i} \int_{U_{i}} \varphi_{i} \Phi_{k} r_{k}^{-m+1} d V_{k 1} d V_{k 2} \\
=\sum_{i} \int_{U_{i}} \varphi_{i} \Phi r^{-m+1} d V_{1} d V_{2}=\int_{M \times M} \Phi r^{-m+1} d V_{1} d V_{2}
\end{gathered}
$$

which establishes Proposition 2.

3. Let $f: M^{m} \rightarrow E^{n}$ be an immersion of a compact oriented differentiable manifold $M^{m}$. Let $H_{n-m-1, n}$ denote the Grassmann manifold of all (unoriented) $(n-m-1)$-planes in $E^{n}$ (it being understood that parallel planes are not identified), and $\left|d H_{n-m-1, n}\right|$ the Euclidean invariant measure on $H_{n-m-1, n}$ (this is worked out explicitly in [6]). Consider the quantity

$$
\mathscr{A}\left(M^{m}\right)=K_{m, n} \int_{H_{n-m-1, n}} \lambda^{2}\left|d H_{n-m-1, n}\right|,
$$

where $\pm \lambda(h)= \pm \lambda\left(h, M^{m}\right)$ denotes the linking number of $h \in H_{n-m-1, n}$ with $M^{m}, K_{m, n}=k_{m, m+2} k_{m, m+3} \cdots k_{m, n}$, and

$$
k_{m, j}=\pi^{-(m+1) / 2} \frac{\Gamma((j+1) / 2)}{\Gamma((j-m) / 2)} .
$$

In particular $K_{m, m+1}=1$, so that $\mathscr{A}\left(M^{m}\right)$ generalizes the volume bounded by a simple closed hypersurface. Furthermore, as is proved in [6, pp. 1341-1342] $\mathscr{A}\left(M^{m}\right)$ is stable under raising of the codimension, that is, if $E^{n}$ is regarded as an $n$-plane in $E^{N}$ so that $f\left(M^{m}\right) \subset E^{n} \subset E^{N}$, then 


$$
K_{m, N} \int_{H_{N-m-1, N}} \lambda^{2}\left|d H_{N-m-1, N}\right|=K_{m, n} \int_{H_{n-m-1, n}} \lambda^{2}\left|d H_{n-m-1, n}\right| .
$$

The proof given in [6] has a slight error, however, which affects the constant, so we give a corrected version here.

Let $H$ denote a family of $(n-m)$-planes in $E^{n+1}$ and let $h=H \cap E^{n} \subset E^{n+1}$. To each $(n-m)$-plane assign an orthonormal frame $X b_{1} \cdots b_{n+1}$ such that $X \in h, b_{1}, \cdots, b_{n-m-1}$ span $h, b_{n-m}$ in $H$ perpendicular to $h$, and $b_{n-m+2}, \cdots$, $b_{n+1} \perp h$ in $E^{n}$. Let $X a_{1} \cdots a_{n+1}$ be another family of frames such that $a_{i}=b_{i}$, $1 \leq i \leq n-m-1, a_{\alpha}=b_{\alpha}, n-m+2 \leq \alpha \leq n+1$, (we use these ranges of $i, \alpha$ until further notice), $a_{n-m}$ is along the orthogonal projection of $b_{n-m}$ into $E^{n}, a_{n-m+1}$ is the unit normal to $E^{n}$ in $E^{n+1}$, so that $a_{n-m+1}$ is constant. We may write

$$
\begin{aligned}
b_{n-m} & =\cos \varphi a_{n-m}+\sin \varphi a_{n-m+1}, \\
b_{n-m+1} & =-\sin \varphi a_{n-m}+\cos \varphi a_{n-m+1} .
\end{aligned}
$$

If we let $d X \cdot a_{i}=\pi_{i}, d X \cdot b_{i}=\rho_{i}, d a_{i} \cdot a_{j}=\pi_{i j}, d b_{i} \cdot b_{j}=\rho_{i j}$, then we have

$$
\begin{aligned}
\rho_{n-m+1} & =-\sin \varphi \pi_{n-m}, \\
\rho_{n-m, \alpha} & =\cos \varphi \pi_{n-m, \alpha}, \\
\rho_{i, n-m+1} & =-\sin \varphi \pi_{i, n-m}, \\
\rho_{n-m, n-m+1} & =d \varphi
\end{aligned}
$$

so that

$$
\begin{aligned}
& \left|d H_{n-m, n+1}\right| \\
& \quad=\left|\rho_{n-m+1} \wedge \cdots \wedge \rho_{n+1} \wedge \prod \rho_{i \alpha} \wedge \prod \rho_{n-m, \alpha} \wedge \prod \rho_{i, n-m+1} \wedge \rho_{n-m, n-m+1}\right| \\
& \quad=\left|\sin ^{n-m} \varphi \cos ^{m} \varphi d \varphi \wedge d \Sigma_{m} \wedge d H_{n-m-1, n}\right|
\end{aligned}
$$

where $d \Sigma_{m}=\prod \pi_{n-m, \alpha}$ is the volume element of the unit $m$-sphere $\Sigma_{m}$ perpendicular to $h$ in $E^{n}$. Hence by Fubini's theorem

$$
\begin{aligned}
& \int_{H_{n-m, n+1}} \lambda^{2}\left|d H_{n-m, n+1}\right| \\
& \quad=\int_{0}^{\pi / 2}\left|\sin ^{n-m} \varphi \cos ^{m} \varphi\right| d \varphi \int_{\Sigma_{m}} d \Sigma_{m} \int_{H_{n-m-1, n}} \lambda^{2}\left|d H_{n-m-1, n}\right| .
\end{aligned}
$$

Now 


$$
\begin{aligned}
\int_{0}^{\pi / 2} \mid & \sin ^{n-m} \varphi \cos ^{m} \varphi \mid d \varphi \int_{\Sigma_{m}} d \Sigma_{m} \\
& =\frac{1}{2} \frac{\Gamma\left(\frac{1}{2}(m+1)\right) \Gamma\left(\frac{1}{2}(n-m+1)\right)}{\Gamma\left(\frac{1}{2}(n+2)\right)} \frac{2 \pi^{(m+1) / 2}}{\Gamma\left(\frac{1}{2}(m+1)\right)}=k_{m, n+1}{ }^{-1}
\end{aligned}
$$

This establishes the assertion for $N=n+1$, and the general result follows by induction on $N$.

In considering $\mathscr{A}\left(M^{m}\right)$ the case $m=0$ is special and requires an additional assumption. A compact oriented 0 -manifold is nothing more than a finite set of points, to each of which is assigned a multiplicity \pm 1 . We shall require that the sum of these multiplicities be zero. Thus the simplest case under consideration is that of a pair of points with opposite multiplicities so that here $\mathscr{A}\left(M^{0}\right)$ is essentially the measure of the hyperplanes that meet the line segment joining the two points, which by the generalized Crofton-Cauchy formula is essentially the distance between the two points.

More generally, suppose $M^{0}$ consists of points $x_{1}, \cdots, x_{q}$ with multiplicities $i_{1}, \cdots, i_{q}$. Orient $E^{n}$ and choose an oriented hyperplane $h_{0}$; then orient each hyperplane so that it makes a positive acute angle with $h_{0}$. Of course, this orientation is indeterminate for hyperplanes perpendicular to $h_{0}$, but these form a set of measure zero and so we can neglect them. Each oriented hyperplane divides $E^{n}$ into two half-spaces, one of which is canonically designated the left-hand half-space $h^{+}$, and the other the right-hand half-space $h^{-}$. It is now readily seen that

$$
\lambda\left(h, M^{0}\right)=\sum_{f\left(x_{i}\right) \in h^{+}} i_{i}=-\sum_{f\left(x_{i}\right) \in h^{-}} i_{i}, \quad 1 \leq i \leq q
$$

so that

$$
\begin{aligned}
\int_{H_{n-1}, n} \lambda^{2}\left|d H_{n-1, n}\right| & =-\int_{H_{n-1, n}} \sum_{f\left(x_{i}\right) \in h^{+}} i_{i} \sum_{f(x j) \in h^{-}} i_{j}\left|d H_{n-1, n}\right| \\
& =-\sum_{i, j} i_{i} i_{j} \int_{H_{n-1, n}} F_{i j}\left|d H_{n-1, n}\right|
\end{aligned}
$$

where $F_{i j}(h)=1$ if $f\left(x_{i}\right) \in h^{+}$and $f\left(x_{j}\right) \in h^{-}$, and $F_{i j}(h)=0$ otherwise. But by the generalized Crofton-Cauchy formula,

$$
\int_{H_{n-1, n}}\left(F_{j i}+F_{i j}\right)\left|d H_{n-1, n}\right|=d_{n-1} r\left(x_{i}, x_{j}\right),
$$

where $d_{n-1}=K_{0, n}^{-1}$ equals the volume of the disc bounded by a unit sphere of dimension $n-2$, and $r\left(x_{i}, x_{j}\right)$ is the distance between $f\left(x_{i}\right)$ and $f\left(x_{j}\right)$. Consequently, 


$$
\mathscr{A}\left(M^{0}\right)=K_{0, n} \int_{H_{n-1, n}} \lambda^{2}\left|d H_{n-1, n}\right|=-\frac{1}{2} \sum_{i, j} r\left(x_{i}, x_{j}\right) i_{i} i_{j} .
$$

Theorem 3. $\mathscr{A}$ is reproductive in the sense of Chern [3], i.e., if $q>n-m-1$, then

$$
l_{m, n, q} \int_{H_{q, n}} \mathscr{A}\left(M^{m} \cap H_{q}\right)\left|d H_{q, n}\right|=\mathscr{A}\left(M^{m}\right),
$$

where

$$
l_{m, n, q}=\frac{K_{m, n}}{K_{q-n+m, q}} \frac{\Sigma_{q-n+m} \cdots \Sigma_{0} \Sigma_{n-q-1} \cdots \Sigma_{0}}{\Sigma_{n} \cdots \Sigma_{0}},
$$

and $\Sigma_{j}=\frac{2 \pi^{(j+1) / 2}}{\Gamma((j+1) / 2)}$ is the surface volume of the unit $j$-sphere. In particular,

$$
\mathscr{A}\left(M^{m}\right)=-\frac{1}{2} l_{m, n, n-m} \int_{H_{n-m, n}} \sum_{x_{i}, x_{j}} r\left(x_{i}, x_{j}\right) i_{i} i_{j}\left|d H_{n-m, n}\right|,
$$

where $x_{1}, x_{2}, \ldots$ are the points of intersection of $M^{m}$ with a moving $(n-m)$ plane $H_{n-m}, r\left(x_{i}, x_{j}\right)$ is the Euclidean distance from $x_{i}$ to $x_{j}$, and $i_{i}$ is the intersection number of $H_{n-m}$ (with some orientation) and $M^{m}$ at $x_{i}$.

Proof. Let us observe first that if $h_{n-m-1}$ is a linear space of dimension $n-m-1$ and $h_{q}$ is a linear space of dimension $q$ containing $h_{n-m-1}$, then

$$
\lambda\left(M^{m}, h_{n-m-1}\right)=\lambda\left(M^{m} \cap h_{q}, h_{n-m-1}\right),
$$

where the first linking number is in the sense of submanifolds of $E^{n}$ and the second in the sense of submanifolds of $h_{q}$.

Let us call this configuration $\left(h_{q}, h_{n-m-1}\right), h_{n-m-1} \subset h_{q}$, a banner. The totality of banners forms a differentiable manifold $B$, and has a measure which is invariant under the action of the group of rigid motions of $E^{n}$. This measure is constructed, according to the method of Chern [2], as follows. Let us assign to each banner (locally) a frame $X e_{1} \cdots e_{n}$ so that $X \in h_{n-m-1}, e_{1}, \cdots, e_{n-m-1}$ are parallel to $h_{n-m-1}$, and $e_{n-m}, \cdots, e_{q}$ are parallel to $h_{q}$. Let $d X \cdot e_{i}=\omega_{i}$, $d e_{i} \cdot e_{j}=\omega_{i j}$. The measure is then given by

$$
|d B|=\left|\omega_{n-m} \wedge \cdots \wedge \omega_{n} \wedge \prod_{\substack{1 \leq i \leq n-m-1 \\ n-m \leq j \leq n}} \omega_{i j} \wedge \prod_{\substack{n-m \leq k \leq q \\ q+1 \leq l \leq n}} \omega_{k l}\right|
$$

To prove the theorem we integrate $\lambda^{2}\left(M^{m}, h_{n-m-1}\right)|d B|$ in two ways and equate the results. 
Let us choose a fixed frame $0 i_{1} \cdots i_{n}$ in $E^{n}$, and let $G_{q-n+m+1, m+1}$ denote the Grassmann manifold of $(q-n+m+1)$-spaces through 0 lying in the span of $i_{n-m}, \cdots, i_{n}$. To each $(n-m-1)$-plane $h_{n-m-1}$ in $E^{n}$ assign locally a frame $X a_{1} \cdots a_{n}$ such that $X \in h_{n-m-1}$, and $a_{1}, \cdots, a_{n-m_{-1}}$ are parallel to $h_{n-m-1}$. For each $h_{n-m-1}$ let $G\left(h_{n-m-1}\right)$ denote the rigid motion which takes $0 i_{1} \cdots i_{n}$ to $X a_{1} \cdots a_{n}$. Define a map $H: H_{n-m-1, n} \times G_{q-n+m+1, m+1} \rightarrow B$ by $H\left(h_{n-m-1}, g_{q-n+m+1}\right)=\left(\operatorname{span}\left(h_{n-m-1}, G\left(g_{q-n+m+1}\right)\right), h_{n-m-1}\right)$. Taking frames $X e_{1} \cdots e_{n}$ as before so that $e_{i}=a_{i}, i \leq n-m-1$, we find that

$$
\omega_{n-m} \wedge \cdots \wedge \omega_{n} \wedge \prod_{\substack{1 \leq i \leq n-m-1 \\ n-m \leq j \leq n}} \omega_{i j}=d H_{n-m-1, n}
$$

and $\prod_{\substack{n-m \leq k \leq q \\ q+1 \leq l \leq n}} \omega_{k l}=d G_{q-n+m+1, m+1}+$ terms involving the differentials on $H_{n-m-1, n}$, so that

$$
H^{*}|d B|=\left|d H_{n-m-1, n} \wedge d G_{q-n+m+1, m+1}\right|,
$$

and, by Fubini's theorem,

$$
\int_{\boldsymbol{B}} \lambda^{2}\left(\boldsymbol{M}^{m}, h_{n-m-1}\right)|d \boldsymbol{B}|=\boldsymbol{g}_{m, n, q} \int_{H_{n-m-1, n}} \lambda^{2}\left(\boldsymbol{M}^{m}, h_{n-m-1}\right)\left|d H_{n-m-1, n}\right|,
$$

where $g_{m, n, q}$ is the total volume of $G_{q-n+m+1, m+1}$. To evaluate this we observe that $G_{a, b}=O_{b} / O_{a} \times O_{b-a}$, where $O_{j}$ denotes the orthogonal group in $j$ variables, and $O_{j} / O_{j-1}=S^{j-1}$, the unit sphere of dimension $j-1$, so that the volume $\mathcal{O}_{j}$ of $O_{j}$ is given by

$$
\begin{gathered}
\mathcal{O}_{j}=\Sigma_{j-1} \mathcal{O}_{j-1}=\Sigma_{j-1} \Sigma_{j-2} \cdots \Sigma_{0}, \\
g_{m, n, q}=\frac{\Sigma_{m} \cdots \Sigma_{0}}{\Sigma_{q-n+m} \cdots \Sigma_{0} \Sigma_{n-q-1} \cdots \Sigma_{0}} .
\end{gathered}
$$

Now let us parametrize the banners in another way. To each $h_{q} \subset E^{n}$ let us associate a frame $Y b_{1} \cdots b_{n}$ so that $Y \in h_{q}$, and $b_{1}, \cdots, b_{q}$ are parallel to $h_{q}$. Let $H_{n-m-1, q}$ denote the Grassmann manifold of linear spaces of dimension $n-m-1$ contained in the linear space through $O$ spanned by $i_{1} \cdots i_{q}$, and let $J\left(h_{q}\right)$ denote the rigid motion of $E^{n}$ which takes $O i_{1} \cdots i_{n}$ to $Y b_{1} \cdots b_{n}$. Define a map $K: H_{q, n} \times H_{n-m-1, q} \rightarrow B$ by $K\left(h_{q}, h_{n-m-1}\right)=\left(h_{q}, J\left(h_{q}\right)\left(h_{n-m-1}\right)\right)$, and take frames $X e_{1} \cdots e_{n}$ as before. Then

$$
d H_{q, n}=\omega_{q+1} \wedge \cdots \wedge \omega_{n} \wedge \prod_{\substack{1 \leq i \leq q \\ q+1 \leq j \leq n}} \omega_{i j},
$$




$$
\begin{gathered}
\omega_{n-m} \wedge \cdots \wedge \omega_{q} \wedge \prod_{\substack{1 \leq i \leq n-m-1 \\
n-m \leq j \leq q}} \omega_{i j} \\
=d H_{n-m-1, q}+\text { terms involving the differentials on } H_{q, n} .
\end{gathered}
$$

Hence

$$
K^{*}|d B|=\left|d H_{q, n} \wedge d H_{n-m-1, q}\right|
$$

and by Fubini's theorem and (3.1) we find that

$$
\begin{aligned}
\int_{B} \lambda^{2}\left(M^{m}, h_{n-m-1}\right)|d B| & =\int \lambda^{2}\left(M^{m} \cap h_{q}, h_{n-m-1}\right)|d B| \\
& =\int_{H q, n} K_{q-n+m, q}^{-1} \mathscr{A}\left(M^{m} \cap h_{q}\right)\left|d H_{q, n}\right|,
\end{aligned}
$$

which, together with (3.2) and (3.3), proves the theorem.

Remark. By a similar argument, together with the generalized CroftonCauchy formula, one may show that the $m$-dimensional surface volume $V\left(M^{m}\right)$ of $M^{m}$ is reproductive, i.e.,

$$
p_{m, n, q} \int_{H_{q}, n} V\left(M^{m} \cap h^{q}\right)\left|d H_{q, n}\right|=V(M),
$$

where $p_{m, n, q}=\frac{m \Sigma_{r-1} k_{m-1, m+2} \cdots k_{m-1, n}}{r \sum_{m-1} k_{r-1, r+2} \cdots k_{r-1, q} g_{m-1, n, q}}$ and $r=m-n+q$. This generalizes the generalized Crofton-Cauchy formula.

Our proof of the isoperimetric inequality depends on another formula for $\mathscr{A}\left(M^{m}\right)$. Let $G \subset M^{m} \times M^{m}$ denote the set of all $(x, y)$ such that $f(x) \neq f(y)$, and note that $M^{m} \times M^{m}-G$ is a set of measure zero. Let $l(x, y)$ denote the line joining $f(x)$ and $f(y)$ oriented from $x$ to $y$, so that $l: G \rightarrow H_{1, n}$, the Grassmann manifold of oriented lines in $E^{n}$, and let $e_{1}(x, y)$ denote the unit vector oriented along $l(x, y)$. Let $X(x, y)=f(x), \omega_{1}=d X \cdot e_{1}$ and $d I=d \omega_{1}$. It was shown in [6] that $d I$ comes from an invariant two-form on $\boldsymbol{H}_{1, n}$, and that

$$
(d I)^{m}=m !(-1)^{m(m-1) / 2} r^{-m} \cos \tau \sin \sigma_{1} \sin \sigma_{2} d V_{2} \wedge d V_{1},
$$

where the $d V_{i}$ and $r$ are as in $\S 2$ above, and $\sigma_{i}, \tau$ are certain angles which we shall discuss below.

Theorem 4. $\mathscr{A}\left(M^{m}\right)$ is finite and

$$
\mathscr{A}\left(M^{m}\right)=\frac{(-1)^{1+m(m+1) / 2}}{m ! \Sigma_{m}} \int_{M \times M^{m}} r(d I)^{m} .
$$


Remark. The finiteness does not seem to have an altogether trivial proof. For it is easy to construct a $C^{\infty}$ closed immersed space curve which has arbitrarily high linking numbers with lines. However, it may be shown that any $C^{1}$-immersed hypersurface has bounded winding number with points and any $C^{2}$-immersed space curve with nowhere vanishing curvature has bounded linking number with lines.

Proof. In case $f$ is an embedding this follows from [6, (2.16)] but for the determination of the constant. It remains for us here to show that the proof of (2.16) of [6] is valid in the present more general case and to determine the constant.

In [6, pp. 1329-1330] a certain subset $G_{+} \subset G_{n-m-1, n-1}$ and a differential form $F^{*} d H_{n-m, n}$ on $\left(M^{m} \times M^{m}-D\right) \times G_{+}$are defined, where $D$ is the diagonal. (What we call $M^{m}$ here is called $P$ there and is there of dimension $m+1$.) The definition of $F^{*} d H_{n-m, n}$ is valid in the present case as long as we restrict ourselves to $G \times G_{+}$. Now $F^{*} d H_{n-m, n}$ is the sum of terms of the form

$$
\begin{array}{r}
\omega_{2} \wedge \cdots \wedge \omega_{m+1} \wedge\left(\omega_{1 k_{1}} \wedge \cdots \wedge \omega_{1 k_{m}}\right) \cos \varphi_{1} \cos \varphi_{2} d G_{n-m-1, n-1} \\
2 \leq k_{1}<\cdots<k_{m} \leq n
\end{array}
$$

where $\varphi_{1}$ and $\varphi_{2}$ are certain angles. (Read " $\sum$ " for " $\prod$ " on lines 14 and 16 of p. 1330 of [6].) Now following the argument used to establish (2.3) and (2.4) of [6] we find that

$$
\left|r F^{*} d H_{n-m, n}\right| \leq\left(\begin{array}{c}
n-1 \\
m
\end{array}\right) r^{-m+1} d V_{1} d V_{2}\left|d G_{n-m-1, n-1}\right|
$$

But this is integrable on $G \times G_{+}$by Fubini's theorem and Proposition 2 above. Hence the integrations over the fibre used to establish (2.16) of [6] are valid and lead to finite quantities, and the rest is valid without change.

To determine the sign and constant in (3.6) we observe that both $\mathscr{A}\left(M^{m}\right)$ and [6, p. 1341] $\int r(d I)^{m}$ are stable under raising of the codimension. Hence the sign and constant are stable and can be found by checking the formula for a sphere $S^{m} \subset E^{m+1}$. The sign is checked using (3.5) and the facts that $\cos \tau$ $=-1$, and $\sin \sigma_{i} \geq 0$ for the sphere. To evaluate the constant now, we use the fact that $\pm(m !)^{-1}(d I)^{m}$ is essentially the invariant measure for oriented lines in $E^{m+1}$; and for each oriented line $L$ meeting $S^{m}$ there is a unique ordered pair of points $(x, y) \in S^{m} \times S^{m}$ such that $l(x, y)=L$, so that the integration can be made on $\boldsymbol{H}_{1, m+1}$. To each oriented line in $E^{m+1}$ we associate a frame $X e_{1} \cdots e_{m+1}$ such that $e_{1}$ is directed along the line and $X$ is a point on the line. Let $d X \cdot e_{i}=\omega_{i}, d e_{i} \cdot e_{j}=\omega_{i j}$. Then $(m !)^{-1}(d I)^{m}= \pm \omega_{2} \wedge \cdots \wedge \omega_{m+1} \wedge \omega_{12} \wedge$ $\cdots \wedge \omega_{1 m+1}$. If the direction of the line is held fixed, $\omega_{2} \wedge \cdots \wedge \omega_{m+1}$ becomes the volume element on a perpendicular $m$-plane, so that 


$$
\begin{aligned}
\int_{L \cap S m \neq 0} r \omega_{2} \wedge \cdots \wedge \omega_{m+1} \wedge \omega_{12} \wedge \cdots \wedge \omega_{1 m+1} \\
\quad=\int \mathscr{A}\left(S^{m}\right) \omega_{12} \wedge \cdots \wedge \omega_{1 m+1}=\Sigma_{m} \mathscr{A}\left(S^{m}\right)
\end{aligned}
$$

which completes the proof of the theorem.

4. In this section we prove Theorem 1. Basic to the proof is an integral formula obtained by integration over a certain "secant space". We begin by defining this space.

Let $M^{m}=M$ be a differentiable manifold of class $C^{2}$ and dimension $m$. Let $S(M)$ denote the two-fold cartesian product of $M$ with the diagonal replaced by its bundle of oriented normal directions. This space, which is fully explained in [6], is a differentiable manifold, with boundary, of class $C^{1}$ and has the universal property that if $f: M^{m} \rightarrow E^{n}$ is an embedding, then there is an induced smooth map $L_{f}: S(M) \rightarrow H_{1, n}$ which assigns to each $(x, y) \in M \times M, x \neq y$, the line directed from $f(x)$ to $f(y)$, and to the boundary points of $S(M)$ the corresponding tangent lines of $M$. Let $\pi: S(M) \rightarrow M \times M$ be the canonical projection map, and $\pi_{i}: M \times M \rightarrow M$ be the projection into the $i$-th factor, $i=1,2$.

Let us now assume that $M$ is compact and oriented with $1 \leq m<n$. For $z \in S(M)$ let $e_{1}(z)$ denote the unit vector in $E^{n}$ directed along $L_{f}(z)$. Let $X(z)=$ $f \pi_{1} \pi(z)$ and $\omega_{1}=d X \cdot e_{1}$, which is a differential 1-form on $S(M)$. Denote $d \omega_{1}$ by $d I$, and the (absolute) euclidean distance from $f \pi_{1} \pi(z)$ to $f \pi_{2} \pi(z)$ by $r(z)$. Consider the differential form $r \omega_{1} \wedge(d I)^{m-1}$. The orientation of $M$ induces an orientation on $M \times M$ and hence on $S(M)$. By applying Stokes' theorem we find that

$$
\int_{T(M)} r \omega_{1} \wedge(d I)^{m-1}=\int_{S(M)} d r \wedge \omega_{1} \wedge(d I)^{m-1}+\int_{S(M)} r(d I)^{m} .
$$

Now the left-hand term is zero, since $r=0$ on $T(M)$. Also, $S(M)$ and $M \times M$ differ by sets of measure zero. Hence we may write

$$
-\int_{M \times M} d r \wedge \omega_{1} \wedge(d I)^{m-1}=\int_{M \times M} r(d I)^{m} .
$$

Let us now give a local analysis of these differential forms. $r(d I)^{m}$ has been analyzed in [6, pp. 1324-1326], and our analysis of the other follows the same procedure and uses the same frames. It is more convenient to give a separate analysis of the case $m=1$. Let $(x, y) \in M \times M$ be such that $f(x) \neq f(y)$ and that the tangent spaces to $f$ at $x$ and $y$ are in general position with respect to $L_{f}(x, y)$ and are not perpendicular to $L_{f}(x, y)$. (All other points of $S(M)$ form a set of measure zero which we ignore.) We now drop the requirement that $f$ be an embedding. 
In case $m=1$, let $a_{2}, b_{2}$ denote the unit tangent vectors to $f$ at $x$ and $y$ respectively such that $e_{1}$ makes positive acute angles $\sigma_{1}, \sigma_{2}$ with either, and let $X=$ $f(x), Y=f(y)$. Then $d X=\pi_{2} a_{2}, d Y=\rho_{2} b_{2}$, and $\omega_{1}=d X \cdot e_{1}=\cos \sigma_{1} \pi_{2}$. Now $(Y-X)=r e_{1}$, and $d r=d\left(r e_{1}\right) \cdot e_{1}$, so that

$$
d r=\cos \sigma_{2} \rho_{2}-\cos \sigma_{1} \pi_{2}, \quad d r \wedge \omega_{1}=\left(\cos \sigma_{1} \cos \sigma_{2}\right) \rho_{2} \wedge \pi_{2} .
$$

We set $\cos \nu=1$ if $a_{2}$ and $b_{2}$ are both positively directed or both negatively directed tangent vectors to our oriented curve, and $\cos \nu=-1$ if one of $a_{2}, b_{2}$ is positively directed and the other negatively directed. From this we get

$$
d r \wedge \omega_{1}=-\left(\cos \nu \cos \sigma_{1} \cos \sigma_{2}\right) d s_{1} \wedge d s_{2},
$$

where $d s_{1}, d s_{2}$ denote the positively directed elements of arc at $x$ and $y$ respectively. By [6] we can also write

$$
r d I=-\left(\cos \tau \sin \sigma_{1} \sin \sigma_{2}\right) d s_{1} \wedge d s_{2},
$$

where $\cos \tau$ is the angle between the 2-planes spanned by $e_{1} a_{2}$ and $e_{1} b_{2}$ with orientations $e_{1} t_{1}$ and $e_{1} t_{2}$, where $t_{1}$ and $t_{2}$ are the positively directed unit tangent vectors to $f$ at $x$ and $y$, respectively.

If $m>1$, let $T_{1}$ denote the (oriented) tangent space to $f$ at $x$, and $S_{1}$ the linear space of dimension $m+1$ spanned by $T_{1}$ and $L_{f}(x, y)$ with orientation $e_{1} T_{1}$. Let $Q_{1}$ be the 2-plane spanned by $e_{1}$ and its orthogonal projection on $T_{1}$. Choose frames $X a_{1} \cdots a_{n}$ so that $a_{1} \perp T_{1}$ in $S_{1}, a_{2}$ in $Q_{1}$, and $a_{3}, \cdots, a_{m+1}$ in $T_{1}$, and by reversing $a_{2}$ or $a_{3}$ if necessary we arrange that $a_{1} \cdots a_{m+1}$ agrees with the orientation $e_{1} T_{1}$, and $a_{2} \cdots a_{m+1}$ agrees with the orientation of $T_{1}$, and that $e_{1}$ and $a_{2}$ make a positive acute angle $\sigma_{1}$. Let $d X \cdot a_{i}=\pi_{i}$. Then

$$
\omega_{1}=d X \cdot e_{1}=\cos \sigma_{1} \pi_{2} \text {. }
$$

Let $T_{2}$ denote the oriented tangent space to $f$ at $y, S_{2}$ the $(m+1)$-plane spanned by $T_{2}$ and $L_{f}(x, y)$ with orientation $e_{1} T_{2}$, and $Q_{2}$ the 2-plane containing $L_{f}(x, y)$ and its orthogonal projection on $T_{2}$. Let us take another family of frames $Y b_{1} \cdots b_{n}$ such that $Y=f(y), b_{1} \perp T_{2}$ in $S_{2}, b_{2}$ in $Q_{2}$, and $b_{3}, \cdots, b_{m+1}$ in $T_{2}$, and by reversing $b_{2}$ or $b_{3}$ if necessary we arrange that $b_{1} \cdots b_{m+1}$ agrees with the orientation $e_{1} T_{2}$, and $b_{2} \cdots b_{m+1}$ agrees with the orientation of $T_{2}$, and that $e_{1}$ and $b_{2}$ make a positive acute angle $\sigma_{2}$. Let $d Y \cdot b_{i}=\rho_{i}$. Then

$$
d r=d\left(r e_{1}\right) \cdot e_{1}=\cos \sigma_{2} \rho_{2}-\cos \sigma_{1} \pi_{2},
$$

whence

$$
d r \wedge \omega_{1}=-\left(\cos \sigma_{1} \cos \sigma_{2}\right) \pi_{2} \wedge \rho_{2} .
$$

Now from the computation in [6, pp. 1324-1326] it follows that 


$$
\begin{aligned}
(d I)^{m-1} \equiv(m-1) !( & -1)^{(m-1)(m-2) / 2} r^{-m+1} \cos \nu \rho_{3} \wedge \cdots \\
& \wedge \rho_{m+1} \wedge \pi_{3} \wedge \cdots \wedge \pi_{m+1}, \quad \bmod \rho_{2}, \pi_{2},
\end{aligned}
$$

where $\cos \nu=a_{3} \wedge \cdots \wedge a_{m+1} \cdot b_{3} \wedge \cdots \wedge b_{m+1}$. From (4.5) and (4.6) we obtain

$$
\begin{aligned}
d r & \wedge \omega_{1} \wedge(d I)^{m-1} \\
& =(m-1) !(-1)^{m(m-1) / 2} r^{-m+1} \cos \nu \cos \sigma_{1} \cos \sigma_{2} d V_{2} \wedge d V_{1},
\end{aligned}
$$

where $d V_{1}, d V_{2}$ denote the elements of volume on $M$ at $x, y$, respectively. Note that (4.7) agrees with (4.2), so that we need not further distinguish the case $m=1$, except when we interpret $\nu$. Let us recall [6] that

$$
r(d I)^{m}=m !(-1)^{m(m-1) / 2} r^{-m+1} \cos \tau \sin \sigma_{1} \sin \sigma_{2} d V_{2} \wedge d V_{1},
$$

where $\tau$ is the angle between the oriented planes $S_{1}$ and $S_{2}$.

Proposition 5. Let $f: M^{m} \rightarrow E^{n}$ be an immersion of class $C^{2}$. Then

$$
\begin{aligned}
-\Sigma_{m} \mathscr{A}\left(M^{m}\right) & =\int_{M \times M} r^{-m+1} \cos \tau \sin \sigma_{1} \sin \sigma_{2} d V_{1} d V_{2} \\
& =-\frac{1}{m} \int_{M \times M} r^{-m+1} \cos \nu \cos \sigma_{1} \cos \sigma_{2} d V_{1} d V_{2} .
\end{aligned}
$$

Proof. For $m=1$ the proof is simple. On $S(M)$ we consider the form $d X \cdot(Y-X)$. Where $e_{1}$ is defined, i.e., for $(x, y) \in M \times M$ such that $f(x) \neq f(y)$, $d X \cdot(Y-X)=r \omega_{2}$. Hence by (4.1) we obtain

$$
-\int_{S(M)} d r \wedge \omega_{1}=\int_{S(M)} r d I .
$$

The second equality follows from this, together with (4.7) and (4.8), while the first follows from Theorem 4 and (4.8).

For general $m$ the first equality follows also from Theorem 4 and (4.8); the second from (4.1), (4.7) and (4.8) provided that $f$ is an embedding. If $f$ is not an embedding, we consider $E^{n}$ as contained in $E^{N}$, where $N \geq 2 n+2$. By the Thom transversality theorem we can find a sequence of embeddings $f_{k}: M \rightarrow E^{N}$ which converge to $f$ uniformly, and whose first derivatives also converge uniformly to those of $f$. Since the second equality holds for $f_{k}$, it holds for $f$ by Proposition 2.

We come now to the proof of Theorem 1 . From the elementary identity

$$
2 \sin ^{2} \frac{1}{2}\left(\sigma_{1}-\sigma_{2}\right)=1-\cos \sigma_{1} \cos \sigma_{2}-\sin \sigma_{1} \sin \sigma_{2}
$$


and Proposition 5, the convergence of the integrals being guaranteed by Proposition 2 , we obtain

$$
\begin{aligned}
& \int_{M \times M} r^{-m+1} 2 \sin ^{2} \frac{1}{2}\left(\sigma_{1}-\sigma_{2}\right) d V_{1} d V_{2} \\
&+\int_{M \times M} r^{-m+1}(1+\cos \tau) \sin \sigma_{1} \sin \sigma_{2} d V_{1} d V_{2} \\
&+\int_{M \times M} r^{-m+1}(1-\cos \nu) \cos \sigma_{1} \cos \sigma_{2} d V_{1} d V_{2} \\
&= \int_{M \times M} r^{-m+1} d V_{1} d V_{2}+\int_{M \times M} r^{-m+1} \cos \tau \sin \sigma_{1} \sin \sigma_{2} d V_{1} d V_{2} \\
&-\int_{M \times M} r^{-m+1} \cos \nu \cos \sigma_{1} \cos \sigma_{2} d V_{1} d V_{2} \\
&= \int_{M \times M} r^{-m+1} d V_{1} d V_{2}-(1+m) \Sigma_{m} \mathscr{A}(M) .
\end{aligned}
$$

Now the expression (4.9) is nonnegative, since $\sigma_{1}$ and $\sigma_{2}$ are acute and positive almost everywhere. Hence we have

$$
\int_{M \times M} r^{-m+1} d V_{1} d V_{2}-(1+m) \Sigma_{m} \mathscr{A}(M) \geq 0 .
$$

Equality holds if and only if $\sigma_{1} \equiv \sigma_{2}, \tau \equiv \pi$, and $\nu \equiv 0$. This holds for a sphere, and in fact for several coincident spheres with coincident orientations or, in case $m=1$, for one or several coincident circles each gone around in the same direction any number of times. Suppose it holds for $f: M \rightarrow E^{n}$. Then let $p \in M$ and let $T_{p}$ denote the tangent $m$-plane to $f$ at $p$. Consider all $m$-spheres in $E^{n}$ tangent to $T_{p}$ at $f(p)$. Through any point of $E^{n}-T_{p}$ there passes a unique such sphere and hence their tangent spaces form a field of $m$-planes on $E^{n}-T_{p}$. The conditions $\sigma_{1} \equiv \sigma_{2}, \tau \equiv \pi, \nu \equiv 0$ imply that $f(M) \cap E^{n}-T_{p}$ is an integral submanifold of this field of $m$-planes. Now no component of $M$ can be mapped into $T_{p}$ by $f$, for then this component would not be immersed. Hence each component of $M$ has a point which is mapped into $E^{n}-T_{p}$. By the uniqueness of integral submanifolds, then, each component of $M$ is mapped onto a sphere through $f(p)$ tangent to $T_{p}$ at $p$. But $p \in M$ is arbitrary. Hence $f(M)$ is a single sphere. The condition $\tau \equiv \pi$ implies that the orientations of coincident branches coincide, and the theorem is proven.

5. In this section we use the methods of the preceeding section to prove some additional similar inequalities.

Replacing $r \omega_{1} \wedge(d I)^{m-1}$ by $r^{q} \omega_{1} \wedge(d I)^{m-1}, q \geq 1$, in the proof of Proposition 5 , we obtain 


$$
\begin{aligned}
-q(m-1) ! & \int_{M \times M} r^{-m+q} \cos \nu \cos \sigma_{1} \cos \sigma_{2} d V_{1} d V_{2} \\
& =m ! \int_{M \times M} r^{-m+q} \cos \tau \sin \sigma_{1} \sin \sigma_{2} d V_{1} d V_{2} \\
& =(-1)^{m(m+1) / 2} \int_{M \times M} r^{q}(d I)^{m}
\end{aligned}
$$

Hence

$$
\begin{aligned}
\int_{M \times M} 2 r^{-m+q} & \sin ^{2} \frac{1}{2}\left(\sigma_{1}-\sigma_{2}\right) d V_{1} d V_{2} \\
& +\int_{M \times M} r^{-m+q}(1+\cos \tau) \sin \sigma_{1} \sin \sigma_{2} d V_{1} d V_{2} \\
& +\int_{M \times M} r^{-m+q}(1-\cos \nu) \cos \sigma_{1} \cos \sigma_{2} d V_{1} d V_{2} \\
= & \int_{M \times M} r^{-m+q} d V_{1} d V_{2}+\int_{M \times M} r^{-m+q} \cos \tau \sin \sigma_{1} \sin \sigma_{2} d V_{1} d V_{2} \\
& -\int_{M \times M} r^{-m+q} \cos \nu \cos \sigma_{1} \cos \sigma_{2} d V_{1} d V_{2},
\end{aligned}
$$

so that

$$
\int_{M \times M} r^{-m+q} d V_{1} d V_{2}+(-1)^{m(m+1) / 2} \frac{m+q}{m ! q} \int_{M \times M} r^{q}(d I)^{m} \geq 0,
$$

with equality holding for one or several coincident spheres with coincident orientations, or $(m=1)$ for one or several coincident circles each traversed a number of times in the same direction. For convex hypersurfaces, i.e., for $n=m+1$, the last integral is a familiar object. In fact for $n=m+1$

$$
-\frac{1}{m !}\left|\int_{M \times M} r^{q}(d I)^{m}\right|=\int_{H_{1}, m+1} \sum r^{q}\left(x_{i}, x_{j}\right) i_{i} i_{j} d H_{1, m+1},
$$

where for each line $l \in H_{1, m+1}, x_{i}$ are the points of intersection of $l$ and $f(M)$, and $i_{i}$ is the intersection number of $l$ with $f(M)$ at $x_{i}$. For convex hypersurfaces we have

$$
\frac{1}{m !}\left|\int_{M \times M} r^{q}(d I)^{m}\right|=2 \int_{H_{1}, m+1} r^{q} d H_{1, m+1}
$$

and therefore 


$$
\int_{M \times M} r^{-m+q} d V_{1} d V_{2}-\frac{2(m+q)}{q} \int_{H_{1}, m+1} r^{q} d H_{1, m+1} \geq 0 .
$$

For $m=2, q=2$ we get

$$
A^{2}-4 \int_{H_{1}, 3} r^{2} d H_{1,3} \geq 0,
$$

where $A$ is the area of the surface. By a formula of Herglotz [1, vol. 2, p. 77] we have

$$
V^{2}=\frac{1}{6} \int_{H_{1,3}} r^{4} d H_{1,3}
$$

and therefore, for $m=2, q=4$,

$$
\int_{M \times M} r^{2} d A_{1} d A_{2}-18 V^{2} \geq 0
$$

where $V$ denotes the volume bounded by the surface. These are just samples of the various inequalities which can be obtained by these methods, and of course the last three formulas may be generalized to higher dimensions.

\section{References}

[ 1 ] W. Blaschke, Vorlesungen über Integralgeometrie, Chelsea, New York, 1949.

[2] S. S. Chern, On integral geometry in Klein spaces, Ann. of Math. 43 (1942) 178189.

[3] - - On the kinematic formula in integral geometry, J. Math. Mech. 16 (1966) 101-118.

[4] Arne Pleijel, Zwei kurze Beweise der isoperimetrische Ungleichung, Arch. Math. (Basel) 7 (1956) 317-319.

[5] W. F. Pohl, The self-linking number of a closed space curve, J. Math. Mech. 17 (1968) 975-985.

[6] _- Some integral formulas for space curves and their generalization, Amer. J. Math. 90 (1968) 1321-1345.

[ 7 ] L. A. Santalo, Introduction to integral geometry, Hermann, Paris, 1953. 